\title{
Haemoglobin as a biomarker for clinical outcomes in chronic obstructive pulmonary disease
}

\author{
Aparna Balasubramanian ${ }^{1}$, Robert J. Henderson ${ }^{1}$, Nirupama Putcha ${ }^{1}$, Ashraf Fawzy ${ }^{1}$, Sarath Raju ${ }^{1}$, \\ Nadia N. Hansel ${ }^{1}$, Neil R. Maclntyre ${ }^{2}$, Robert L. Jensen ${ }^{3}$, Gregory L. Kinney $\mathbb{1}^{4}$, William W. Stringer ${ }^{5}$, \\ Craig P. Hersh ${ }^{6}$, Russell P. Bowler ${ }^{7}$, Richard Casaburi ${ }^{5}$, MeiLan K. Han ${ }^{8}$, Janos Porszasz ${ }^{5}$, Barry J. Make $\mathbb{1}^{4}$, \\ Meredith C. McCormack ${ }^{1}$ and Robert A. Wise $\mathbb{1}^{1}$
}

${ }^{1}$ Division of Pulmonary and Critical Care Medicine, Johns Hopkins University, Baltimore, MD, USA. ${ }^{2}$ Division of Pulmonary and Critical Care Medicine, Duke University, Durham, NC, USA. ${ }^{3}$ Division of Pulmonary and Critical Care Medicine, University of Utah, Salt Lake City, UT, USA. ${ }^{4}$ Dept of Epidemiology, Colorado School of Public Health, University of Colorado, Denver, CO, USA. ${ }^{5}$ Los Angeles Biomedical Research Institute at Harbor-UCLA Medical Center, Torrance, CA, USA. ${ }^{6}$ Channing Division of Network Medicine, Brigham and Women's Hospital, Boston, MA, USA. 'Division of Pulmonary, Critical Care and Sleep Medicine, National Jewish Health, Denver, CO, USA. ${ }^{8}$ Division of Pulmonary and Critical Care Medicine, University of Michigan, Ann Arbor, MI, USA.

Corresponding author: Robert Wise (rwise@jhmi.edu)

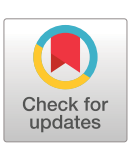

This version is distributed under the terms of the Creative Commons Attribution NonCommercial Licence 4.0. For commercial reproduction rights and permissions contact permissions@ersnet.org

This article has supplementary material available from openres.ersjournals.com

Received: 27 Jan 2021 Accepted: 11 May 2021

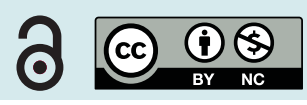

Shareable abstract (@ERSpublications)

Haemoglobin is nonlinearly associated with clinical outcomes in COPD, with increased morbidity at either extreme of the observed range, suggesting that therapeutic correction of anaemia might be most beneficial if targeting normal haemoglobin values https://bit.ly/3ovl7Lt

Cite this article as: Balasubramanian A, Henderson RJ, Putcha N, et al. Haemoglobin as a biomarker for clinical outcomes in chronic obstructive pulmonary disease. ERJ Open Res 2021; 7: 00068-2021 [DOI: 10.1183/23120541.00068-2021].

\section{Abstract}

In COPD, anaemia is associated with increased morbidity, but the relationship between haemoglobin over its entire observed range and morbidity is poorly understood. Such an understanding could guide future therapeutic targeting of haemoglobin in COPD management. Leveraging the COPDGene study, we conducted a cross-sectional analysis of haemoglobin from COPD participants, examining symptoms, quality of life, functional performance, and acute exacerbations of COPD (AECOPD). Haemoglobin was analysed both as a continuous variable and categorised into anaemia, normal haemoglobin, and polycythaemia groups. Fractional polynomial modelling was used for continuous analyses; categorical models were multivariable linear or negative binomial regressions. Covariates included demographics, comorbidities, emphysema, diffusing capacity, and airflow obstruction. From 2539 participants, 366 (14\%) were identified as anaemic and 125 (5\%) as polycythaemic. Compared with normal haemoglobin, anaemia was significantly associated with increased symptoms (COPD Assessment Test score: $\mathrm{p}=0.006$, modified Medical Research Council (mMRC) Dyspnoea Score: $\mathrm{p}=0.001$ ); worse quality of life (St. George's Respiratory Questionnaire (SGRQ) score: $\mathrm{p}<0.001$; Medical Outcomes Study Short Form 36-item Questionnaire (SF-36) General Health: $p=0.002$; SF-36 Physical Health: $p<0.001$ ), decreased functional performance (6-min walk distance (6MWD): $\mathrm{p}<0.001)$, and severe AECOPD ( $\mathrm{p}=0.01$ ), while polycythaemia was not. Continuous models, however, demonstrated increased morbidity at both ends of the haemoglobin distribution $(\mathrm{p}<0.01$ for mMRC, SGRQ, SF-36 Physical Health, 6MWD, and severe AECOPD). Evaluating interactions, both diffusing capacity and haemoglobin were independently associated with morbidity. We present novel findings that haemoglobin derangements towards either extreme of the observed range are associated with increased morbidity in COPD. Further investigation is necessary to determine whether haemoglobin derangement drives morbidity or merely reflects systemic inflammation, and whether correcting haemoglobin towards the normal range improves morbidity.

\section{Introduction}

Derangements in haemoglobin are common in COPD, with prevalence of anaemia and polycythaemia reported as high as $33 \%$ and $6 \%$, respectively [1, 2]. Numerous studies have previously described an association between anaemia and COPD morbidity, including symptoms, quality of life, exercise 
performance, and mortality [3-8]. Further, COPD patients with anaemia have increased healthcare use [9]. As a result, interventions targeting correction of anaemia have been considered to improve outcomes in COPD patients. To that end, however, a deeper understanding of morbidity across the spectrum of haemoglobin values in a COPD population would be a key step towards identifying potential thresholds that optimally demarcate morbidity.

Few studies have examined associations with COPD morbidity across a range of haemoglobin values that spans both anaemia and polycythaemia [2, 6], and to our knowledge prior studies have not evaluated haemoglobin as a continuous variable across its observed range. This is probably related to the varied pathophysiology represented by haemoglobin derangements; anaemia is believed to be related to chronic inflammation [10-12], whereas polycythaemia is often secondary to chronic hypoxaemia. In the era of long-term oxygen therapy, polycythaemia is noted to be less frequent $[13,14]$, and the clinical relevance of polycythaemia is poorly understood. Furthermore, physiological gas transfer interactions with anaemia and polycythaemia as they pertain to morbidity are unknown. Despite these complexities, understanding the association between haemoglobin as a continuous variable across its range and COPD morbidity is an important step in establishing the relevance of a given haemoglobin value in a clinical setting.

This study aims to evaluate the association between haemoglobin and COPD morbidity across the observed range, hypothesising that both anaemia and polycythaemia are associated with increased COPD morbidity. The COPDGene study offers an ideal opportunity to evaluate these associations in a large, well-phenotyped stable COPD cohort.

Methods

Study population and design

COPDGene is a prospective observational study of participants aged 45-80 years with or without prior smoking history conducted across 21 clinical centres. The COPDGene study methodology has been previously reported [15]. Initial enrolment occurred from 2007-2012, with follow-up visits scheduled every 5 years. The present investigation is a cross-sectional study of participants with COPD (defined as forced expiratory volume in $1 \mathrm{~s}\left(\mathrm{FEV}_{1}\right)$ /forced vital capacity $<70 \%$ with $\geqslant 10$ pack-years smoking history) for whom complete blood count (CBC) data at the visit 5 years after original enrolment was available. From the 6284 participants who completed the 5-year visit, 2539 met inclusion criteria (figure 1). COPDGene was approved by institutional review boards at all participating centres. Each participant provided written informed consent.

\section{Anaemia and polycythaemia characterisation}

Haemoglobin concentrations, haematocrit, mean corpuscular volume (MCV), and mean corpuscular haemoglobin concentration were obtained from a CBC drawn at the 5-year visit. Anaemia was defined as a haemoglobin concentration $<12 \mathrm{~g} \cdot \mathrm{dL}^{-1}$ in females, and $<13 \mathrm{~g} \cdot \mathrm{dL}^{-1}$ in males [16], while polycythaemia

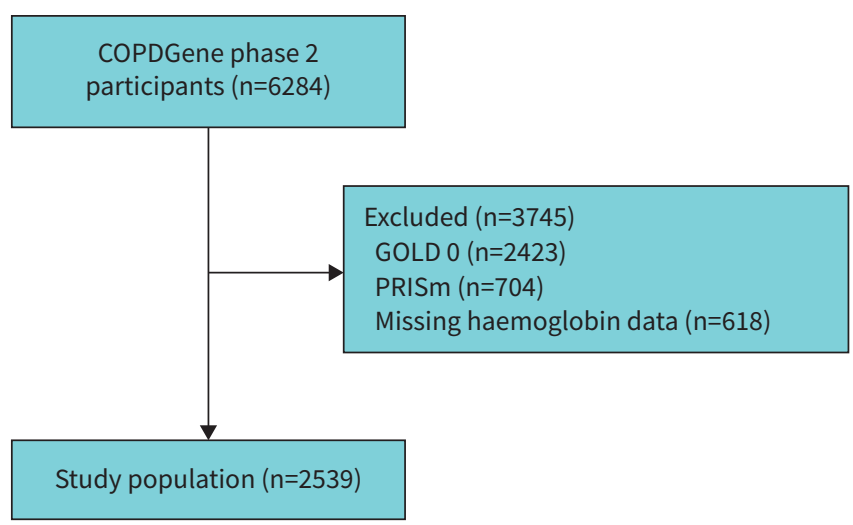

FIGURE 1 Participant selection. Excluded participants include individuals with a forced expiratory volume in $1 \mathrm{~S}\left(\mathrm{FEV}_{1}\right) /$ forced vital capacity ratio $\geqslant 0.7$ who had an $\mathrm{FEV}_{1}>80 \%$ predicted (GOLD 0 ) or had impairment in $\mathrm{FEV}_{1}<80 \%$ predicted (Preserved Ratio Impaired Spirometry, PRISm), and those without available haemoglobin data. Study population included only those with COPD (GOLD 1-4) and haemoglobin data. GOLD: Global Initiative for Chronic Obstructive Lung Disease. 
was defined as haemoglobin $>16 \mathrm{~g} \cdot \mathrm{dL}^{-1}$ in females, and $>16.5 \mathrm{~g} \cdot \mathrm{dL}^{-1}$ in males [17]. Haematocrit values were not included in definitions for the purposes of consistency across analyses, as haemoglobin is the primary measure of interest.

\section{Physiological testing}

Spirometry and diffusing capacity of the lung for carbon monoxide $\left(D_{\mathrm{LCO}}\right)$ measurements were conducted using the ndd EasyOne Pro in accordance with European Respiratory Society (ERS)/American Thoracic Society (ATS) guidelines [18], with standardisation of protocols and quality control procedures across clinical sites. Only subjects with tests judged acceptable and reproducible were included. FEV 1 and $D_{\text {LCO }} \%$ predicted values were calculated using Global Lung Initiative reference equations $[19,20]$, with $D_{\text {LCO }}$ values adjusted for haemoglobin and altitude [20]. Six-minute walk tests (6MWTs) were conducted per ATS guidelines [21].

\section{Computed tomography}

Computed tomography (CT) scans were acquired using individual site CT scanners, with previously published protocols for each scanner type [22]. Total \% emphysema (\%LAA_950) was defined as the per cent of voxels with an attenuation at or below -950 Hounsfield units (HU), quantified using Thirona software (http://www.thirona.eu).

\section{Patient-reported outcomes}

COPD Assessment Test (CAT) [23], St. George’s Respiratory Questionnaire (SGRQ) [24], modified Medical Research Council (mMRC) Dyspnoea Score [25], and Medical Outcomes Study Short Form 36-item Questionnaire (SF-36) [26] were administered and scored to assess quality of life and impact of symptoms.

\section{Exacerbations}

Exacerbation rate was determined from self-reported episodes of increased COPD symptoms requiring antibiotics or steroids in the 12 months preceding this evaluation. Severe exacerbations were defined as the subset of exacerbations requiring an emergency room (ER) visit or hospitalisation, while moderate exacerbations were those that did not.

\section{Statistical analysis}

Participant characteristics were described using means with standard deviations, unless otherwise specified. Prevalence of anaemia and polycythaemia were described by COPD disease severity using the Global Initiative for Chronic Obstructive Lung Disease (GOLD) classification [27]. We analysed morbidity using haemoglobin as both a categorical and continuous variable. For categorical analyses, multivariable linear and negative binomial regression models were constructed for outcomes as appropriate with the normal haemoglobin group set as reference. All models were adjusted for age, sex, ethnicity, education, body mass index (BMI), pack-years smoking history, smoking status, $\mathrm{FEV}_{1} \%$ predicted, $D_{\mathrm{LCO}} \%$ predicted, and \%LAA_950. Further, models were adjusted for self-report of the following comorbidities: congestive heart failure (CHF), hypertension (HTN), diabetes mellitus (DM), and chronic kidney disease (CKD). A sensitivity analysis was performed excluding non-normocytic individuals to address possible differences due to microcytic or macrocytic anaemia. Given the small sample sizes for these groups, formal statistical interaction testing was not performed. A second sensitivity analysis included oxygen use and resting oxygen saturation as covariates to address confounding with regard specifically to the relationship between polycythaemia and morbidity.

For models with haemoglobin treated as a continuous variable, fractional polynomial regression modelling was used to characterise nonlinear relationships. Using the "fp" package (StataCorp), powers for the haemoglobin term were selected from the set $\{-2,-1,-0.5,0,0.5,1,2,3\}$ to identify the best-fitting polynomial model. Point estimates and 95\% confidence intervals displayed in the figures are predicted values for the adjusted associations between haemoglobin and outcomes using the "marginscontplot" package (StataCorp) [28, 29]. Fractional polynomial models were similarly adjusted for the covariates used in the categorical analyses.

We conducted interaction analyses using the “mfpigen” package (StataCorp) [30], which uses fractional polynomial modelling to test and visualise interaction effects between continuous predictors. We explored the unadjusted interaction between haemoglobin and $D_{\text {LCO }} \%$ predicted to evaluate the combined and independent effects of impaired gas transfer and alterations in haemoglobin on morbidity. Our $D_{\text {LCO }} \%$ predicted values were corrected for haemoglobin, to better capture the effects of membrane diffusivity alone and minimise correlations between haemoglobin and $D_{\text {LCO }}$ in analyses. 
All analyses were conducted using Stata 15.1 (StataCorp LLC, College Station, TX, USA) [31], with significance for main effects set at a p-value of $<0.05$ and for interactions set at a p-value $<0.1$.

Results

Participant characteristics

The study population included 2539 participants with COPD GOLD spirometry stage 1-4 with haemoglobin measurements (figure 1). Haemoglobin values in the study cohort ranged from 6.2$19.6 \mathrm{~g} \cdot \mathrm{dL}^{-1}$, with a median (interquartile range (IQR)) of $14(13-15)$. Approximately $14 \%$ of participants were anaemic and 5\% were polycythaemic. Baseline characteristics categorised by clinical definitions of anaemia, normal haemoglobin, and polycythaemia are presented in table 1. A higher percentage of anaemic participants were African American and had cardiovascular and renal comorbidities than those with normal haemoglobin. Polycythaemic participants were over $80 \%$ male, and a majority were current smokers. Approximately $20 \%$ of participants used oxygen in the normal haemoglobin and polycythaemia groups, compared with 34\% of anaemic participants. Average MCV tended to be higher in the normal haemoglobin and polycythaemia groups. When categorised into microcytic (MCV $<80 \mathrm{fL}$ ), normocytic (80-100 fL), and macrocytic (MCV >100 fL), a greater percentage of anaemic participants were microcytic than those with polycythaemia or normal haemoglobin (supplemental table 1). Conversely, polycythaemic participants had a higher percentage with macrocytosis (supplemental table 1). Proteomic analyses of C-reactive protein (CRP), a measure of systemic inflammation, and transferrin, a biomarker reflecting iron

\section{TABLE 1 Participant characteristics}

\begin{tabular}{|c|c|c|c|}
\hline Characteristic & Anaemia & Normal haemoglobin & Polycythaemia \\
\hline Subjects $n$ & 366 & 2048 & 125 \\
\hline Age years & $70 \pm 9$ & $68 \pm 8$ & $66 \pm 8$ \\
\hline Female & $151(41)$ & $956(47)$ & $24(19)$ \\
\hline African American & $131(36)$ & $451(22)$ & $20(16)$ \\
\hline \multicolumn{4}{|l|}{ Education } \\
\hline Eighth grade or less & $12(3)$ & $40(2)$ & $2(2)$ \\
\hline High school, no diploma & $45(12)$ & $177(9)$ & $6(5)$ \\
\hline High school graduate or GED & $93(25)$ & $505(25)$ & $29(23)$ \\
\hline Some college, no degree & $113(31)$ & $561(27)$ & $43(34)$ \\
\hline College or technical degree & $75(20)$ & $548(27)$ & $32(26)$ \\
\hline Master's or Doctoral degree & $28(8)$ & $217(11)$ & $13(10)$ \\
\hline $\mathrm{BMI} \mathrm{kg} \cdot \mathrm{m}^{-2}$ & $29 \pm 7$ & $28 \pm 6$ & $28 \pm 5$ \\
\hline \multicolumn{4}{|l|}{ Smoking status } \\
\hline Former smoker & $267(73)$ & $1321(65)$ & $55(44)$ \\
\hline Current smoker & $99(27)$ & $727(36)$ & $70(56)$ \\
\hline Smoking history pack-years & $53 \pm 26$ & $50 \pm 25$ & $50 \pm 21$ \\
\hline Oxygen use & $123(37)$ & $453(22)$ & $24(19)$ \\
\hline Resting oxygen saturation $\%$ & $95 \pm 4$ & $95 \pm 3$ & $94 \pm 4$ \\
\hline Congestive heart failure & $49(13)$ & $87(4)$ & $5(4)$ \\
\hline Coronary artery disease & $55(15)$ & $221(11)$ & $17(14)$ \\
\hline Hypertension & $252(69)$ & $1069(52)$ & $68(54)$ \\
\hline Diabetes & $81(22)$ & $326(16)$ & $16(13)$ \\
\hline Chronic kidney disease & $34(9)$ & $58(3)$ & $1(1)$ \\
\hline Sleep apnoea & $38(10)$ & $242(12)$ & $15(12)$ \\
\hline $\mathrm{FEV}_{1} \%$ predicted & $57 \pm 22$ & $61 \pm 23$ & $59 \pm 20$ \\
\hline FVC $\%$ predicted & $79 \pm 20$ & $83 \pm 20$ & $80 \pm 18$ \\
\hline $\mathrm{FEV}_{1} / \mathrm{FVC}$ ratio & $0.53 \pm 0.13$ & $0.54 \pm 0.12$ & $0.55 \pm 0.12$ \\
\hline$D_{\text {Lco }} \%$ predicted & $62 \pm 22$ & $67 \pm 22$ & $67 \pm 23$ \\
\hline \%LAA_-950, median (IQR) & $5(2-16)$ & $6(2-16)$ & $5(2-12)$ \\
\hline LAA $_{-950}>5 \%{ }^{\#}$ & $159(51)$ & $994(53)$ & $55(48)$ \\
\hline MCV fL & $89 \pm 8$ & $92 \pm 5$ & $94 \pm 4$ \\
\hline \multicolumn{4}{|c|}{ 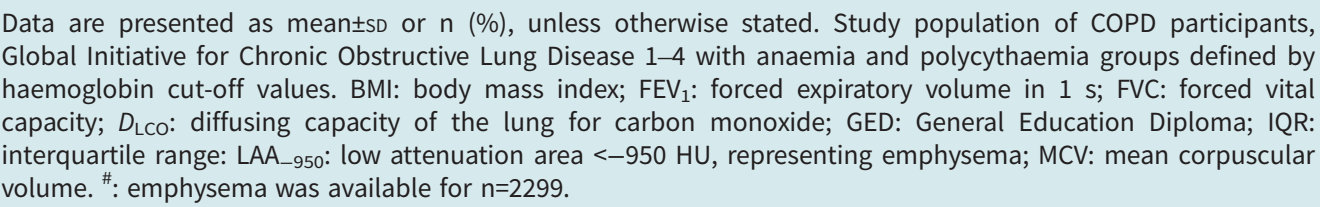 } \\
\hline
\end{tabular}


stores, demonstrated higher CRP among anaemic participants than participants with normal haemoglobin (supplemental figure 1). No differences were noted across groups in transferrin.

The study population had on average moderate airflow obstruction, with all three groups demonstrating similar mean $\mathrm{FEV}_{1} \%$ predicted values $(57 \%, 61 \%$, and $59 \%$ for anaemic, normal, and polycythaemic participants, respectively). Gas transfer, after correction for haemoglobin and altitude, tended to be more impaired among the anaemic participants ( $D_{\text {LCO }} 62 \%$ predicted) compared to normal and polycythaemic participants ( $D_{\text {LCO }} 67 \%$ predicted). The three groups had similar degrees of emphysema, with roughly half of all participants demonstrating greater than $5 \%$ low attenuation areas on CT.

Using the GOLD classification of disease severity [27], we observed increasing anaemia prevalence with greater COPD disease severity. In contrast, no clear pattern of polycythaemia prevalence emerges with disease severity (supplemental figure 2).

\section{Anaemia is independently associated with COPD morbidity}

Multivariable models comparing anaemic participants to those with normal haemoglobin demonstrated independent associations with symptoms, quality of life, and functional performance by 6-min walk distance (6MWD) (table 2). Anaemic participants demonstrated on average an SGRQ score 4.19 points (95\% CI 1.86-6.51, $\mathrm{p}<0.001$ ) higher than those with normal haemoglobin; compared to a minimum clinically important difference (MCID) of 4 [32], this association is both statistically and clinically significant. Similarly, on the 6MWT, anaemic participants walked on average $51 \mathrm{~m}$ less than normal haemoglobin participants (95\% CI 36.6-65.4, $\mathrm{p}<0.001$ ), which is again a clinically relevant difference compared to an MCID of $25 \mathrm{~m}$ [33, 34]. In addition, anaemia was associated with a 63\% higher rate of severe exacerbations (rate ratio (RR) 1.63, 95\% CI 1.1-2.4, p=0.01) as compared with normal haemoglobin (table 2). In sensitivity analyses excluding individuals with microcytosis or macrocytosis, similar results were observed although anaemia no longer demonstrated statistically significant associations with CAT score, SF-36 mental health component score, or severe exacerbations (supplemental table 2).

There were no significant associations between polycythaemia and morbidity (table 2) compared with normal haemoglobin. Polycythaemia tended towards higher rates of severe exacerbations (RR 1.24, 95\% CI 0.64-2.38, $\mathrm{p}=0.52$ ) but did not achieve statistical significance for either moderate or severe exacerbations (table 2). Additional sensitivity analyses adjusting for resting oxygen saturation and long-term oxygen use, demonstrated similar results (supplemental table 3).

\section{Haemoglobin demonstrates a nonlinear association with clinical morbidity}

Examining haemoglobin as a continuous variable using flexible modelling methods provided evidence of nonlinear relationships between haemoglobin and COPD morbidity across the observed range (figure 2). Specifically, morbidity was increased at both extremes of the range of haemoglobin values for most

\begin{tabular}{|c|c|c|c|c|}
\hline & \multicolumn{2}{|c|}{ Anaemia } & \multicolumn{2}{|c|}{ Polycythaemia } \\
\hline & $\boldsymbol{\beta}(95 \% \mathrm{Cl})^{\#}$ & p-value & $\boldsymbol{\beta}(95 \% \mathrm{Cl})^{\#}$ & p-value \\
\hline CAT score & $1.40(0.40-2.41)$ & 0.006 & $-0.31(-1.80-1.19)$ & 0.69 \\
\hline mMRC dyspnoea score & $0.28(0.12-0.44)$ & 0.001 & $0.07(-0.16-0.31)$ & 0.55 \\
\hline SGRQ score & $4.19(1.86-6.51)$ & $<0.001$ & $0.49(-2.95-3.94)$ & 0.78 \\
\hline SF-36 general & $-2.14(-3.47--0.81)$ & 0.002 & $1.24(-0.73-3.21)$ & 0.22 \\
\hline SF-36 physical function & $-2.80(-4.13--1.48)$ & $<0.001$ & $0.40(-1.57-2.37)$ & 0.69 \\
\hline SF-36 mental health & $-1.47(-2.92--0.01)$ & 0.048 & $-0.37(-2.52-1.79)$ & 0.74 \\
\hline 6MWD m & $-51.0(-65.4--36.6)$ & $<0.001$ & $-0.2(-21.4-20.9)$ & 0.98 \\
\hline Moderate exacerbations ${ }^{\#}$ & $1.06(0.76-1.49)$ & 0.72 & $0.71(0.41-1.24)$ & 0.23 \\
\hline Severe exacerbations ${ }^{\#}$ & $1.63(1.10-2.40)$ & 0.01 & $1.24(0.64-2.38)$ & 0.52 \\
\hline \multicolumn{5}{|c|}{$\begin{array}{l}\text { Models adjusted for age, sex, ethnicity, education, body mass index, pack-years smoking, smoking status, } \\
\text { congestive heart failure, hypertension, diabetes mellitus, chronic kidney disease, \% emphysema, forced } \\
\text { expiratory volume in } 1 \mathrm{~s} \% \text { predicted, and diffusing capacity of the lung for carbon monoxide \% predicted. } \\
\text { Coefficients and p-values are in comparison to normal haemoglobin. } \beta \text { coefficient units are points for CAT, } \\
\text { mMRC, SGRQ, and SF-36 scores, and metres for 6MWD. CAT: COPD Assessment Test; mMRC: modified Medical } \\
\text { Research Council Dyspnoea Score; SGRQ: St. George's Respiratory Questionnaire; SF-36: Medical Outcomes } \\
\text { Study Short Form 36-item Questionnaire; 6MWD: 6-min walk distance. \#: RR (95\% CI) for moderate and severe } \\
\text { exacerbations. }\end{array}$} \\
\hline
\end{tabular}



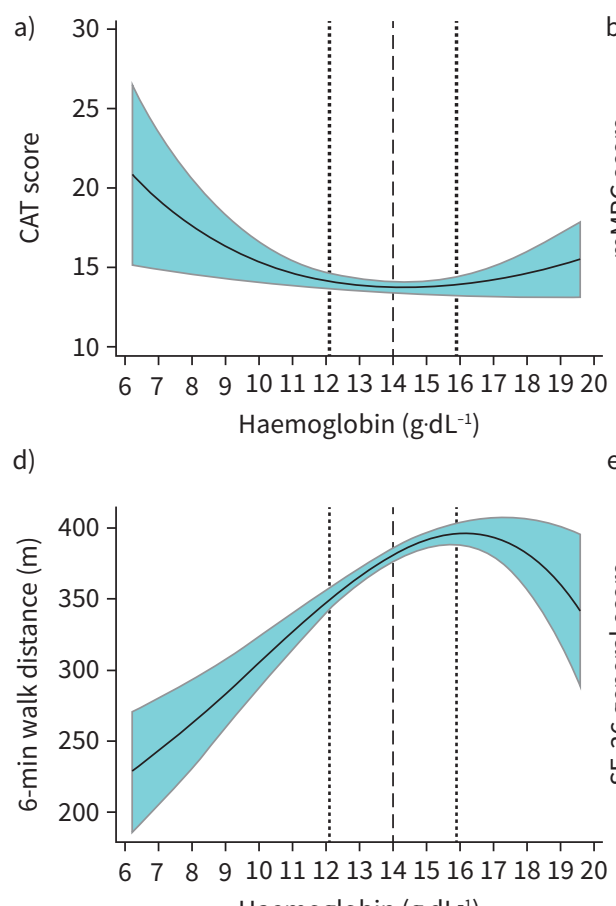
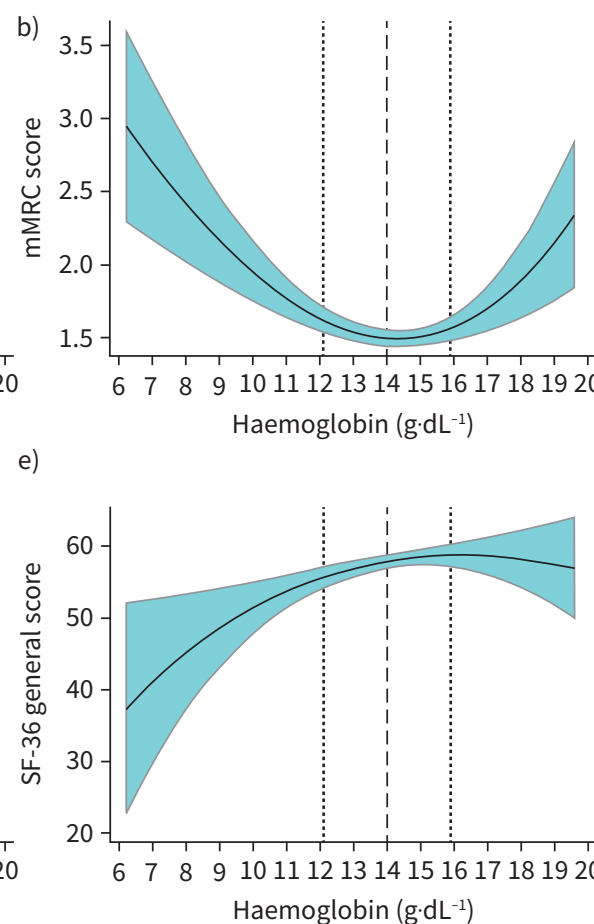

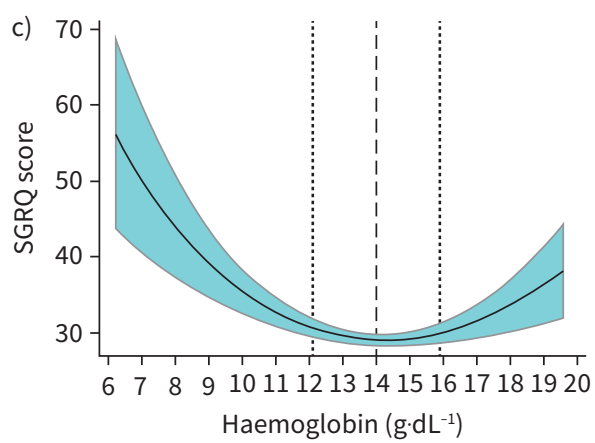

f)

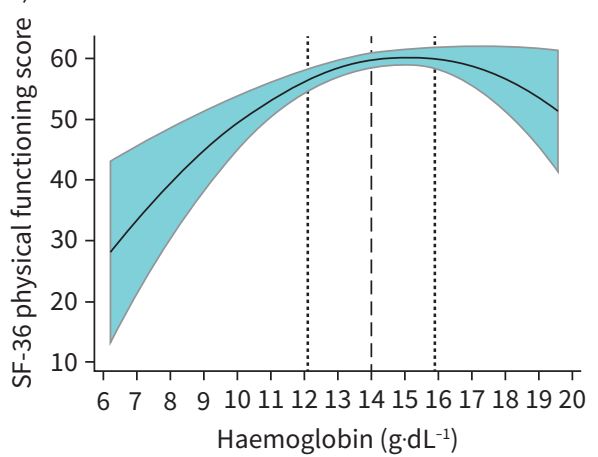

FIGURE 2 Morbidity across the range of haemoglobin values: a) COPD Assessment Test (CAT), b) modified Medical Research Council Dyspnoea Score (mMRC), c) St. George's Respiratory Questionnaire (SGRQ), d) 6-min walk distance, and e, f) Medical Outcomes Study Short Form 36-item Questionnaire (SF-36). Models adjusted for age, sex, ethnicity, education, pack-years smoked, smoking status, body mass index, congestive heart failure, hypertension, diabetes mellitus, chronic kidney disease, forced expiratory volume in $1 \mathrm{~s} \%$ predicted, diffusing capacity of the lung for carbon monoxide \% predicted, and \% emphysema. Dashed line represents median $\left(14 \mathrm{~g} \cdot \mathrm{dL}^{-1}\right)$, and dotted lines represent 10 th $\left(12.1 \mathrm{~g} \cdot \mathrm{dL}^{-1}\right)$ and 90 th $\left(15.9 \mathrm{~g} \cdot \mathrm{dL}{ }^{-1}\right)$ percentiles of haemoglobin. 
outcomes. This pattern was most evident with mMRC dyspnoea score, SGRQ score, 6MWD, and SF-36 physical functioning score (figure $2 \mathrm{~b}-\mathrm{d}, \mathrm{f}$ ). In contrast with the categorical analyses, participants in both the anaemia and polycythaemia ranges had increased symptoms, decreased quality of life, and reduced functional performance.

Moderate exacerbations tended to be stable across the observed range of haemoglobin values, while severe exacerbations were increased among severely anaemic and polycythaemic participants (supplemental figure 3). Statistical testing of the combined haemoglobin terms in the fractional polynomial models for all outcomes are presented in supplemental table 4.

\section{$\mathrm{D}_{L C O}$ does not modify the association between haemoglobin and morbidity}

To evaluate the interrelated physiological processes of anaemia and gas transfer, we examined the interaction between $D_{\mathrm{LCO}}$ and haemoglobin on morbidity. Of note, $D_{\mathrm{LCO}} \%$ predicted was corrected for haemoglobin, to better capture diffusivity specifically. The association between haemoglobin and morbidity was found to be consistent across a wide range of $D_{\text {LCO }}$ values, and with greater $D_{\text {LCO }}$ impairment, there was a consistent increase in morbidity across all outcomes (figure). Significant interactions were identified between $D_{\mathrm{LCO}}$ and haemoglobin for mMRC (figure $3 \mathrm{~b}$ ) and SGRQ score (figure 3c), but not for CAT score, 6MWD, SF-36 (figure 3a, $\mathrm{d}-\mathrm{f}$ ), moderate exacerbations ( $\mathrm{p}=0.94$ ), or severe exacerbations $(p=0.21)$ (exacerbation plots not shown). For mMRC and SGRQ, the effect of haemoglobin on morbidity is attenuated below a $D_{\text {LCO }}$ of $\sim 30 \%$ predicted, with minimal variation in morbidity scores across the haemoglobin range. In other words, with very severe reduction in $D_{\mathrm{LCO}}$, breathlessness is high, and quality of life is poor, irrespective of haemoglobin value.

\section{Discussion}

In this well-characterised cohort of COPD participants, haemoglobin derangements (anaemia or polycythaemia) were found to be prevalent (19\%) and associated with increased morbidity including symptoms, quality of life, functional exercise performance, and hospitalisations for COPD exacerbations. We demonstrate that clinical categorisation of anaemia and polycythaemia incompletely describes the
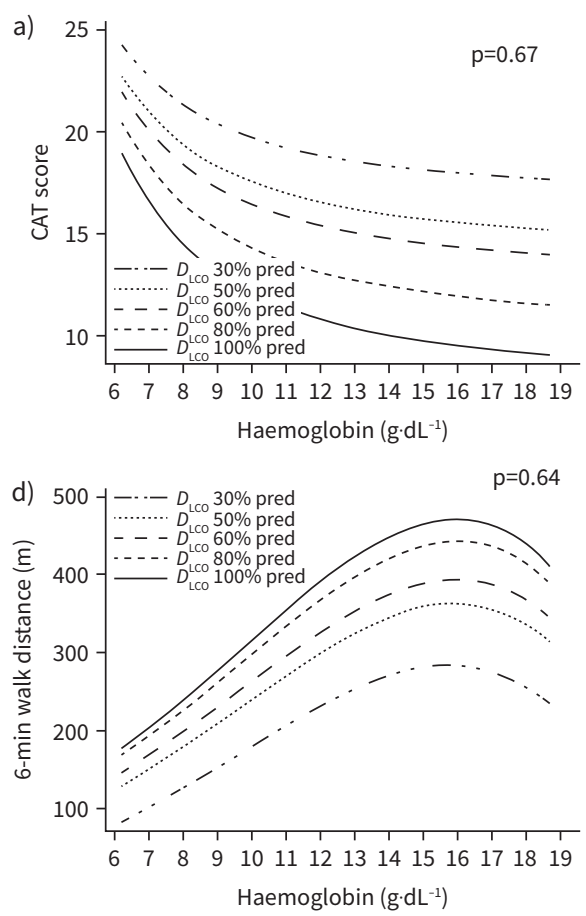

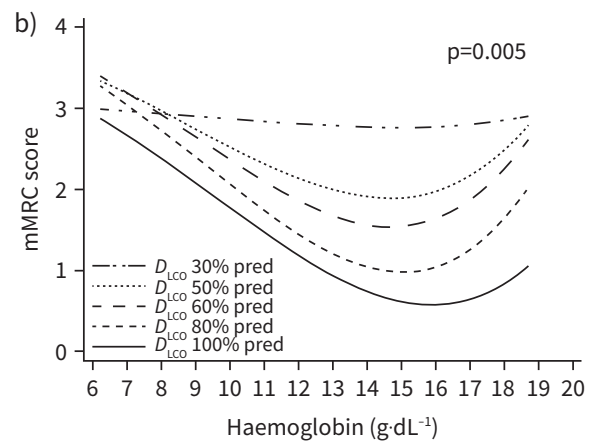

Haemoglobin $\left(\mathrm{g} \cdot \mathrm{dL}^{-1}\right)$

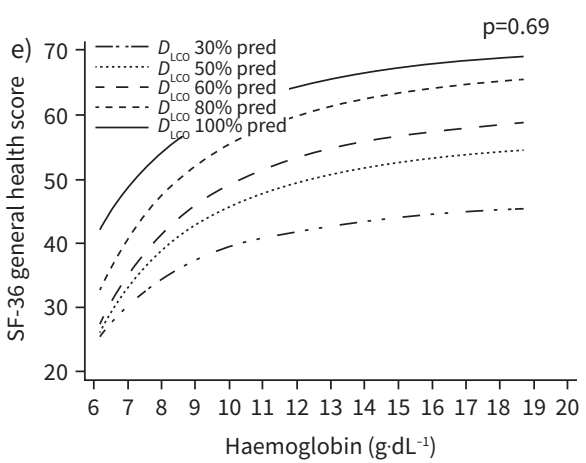

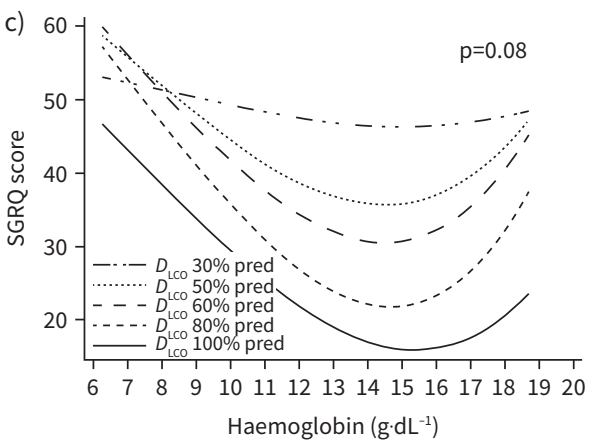

f)

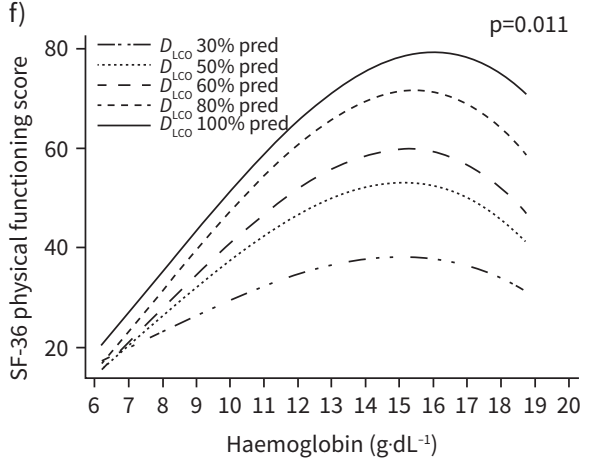

FIGURE 3 Relationship between haemoglobin and COPD outcomes by level of gas transfer impairment. a) COPD Assessment Test (CAT), b) modified Medical Research Council Dyspnoea Score (mMRC), c) St. George's Respiratory Questionnaire (SGRQ), d) 6-min walk distance, e) Medical Outcomes Study Short Form 36-item Questionnaire (SF-36) general health score and f) SF-36 physical functioning score. Models were unadjusted regressions of clinical outcomes on the interaction between haemoglobin and diffusing capacity of the lung for carbon monoxide $\left(D_{\mathrm{Lco}}\right) \%$ predicted. Plots are for marginal predictions at $D_{\text {LCO }} \%$ predicted values of $30,50,60,80$, and $100 \%$ predicted. $D_{\text {LCo }} \%$ predicted is adjusted for altitude and haemoglobin. p-values correspond to the interaction term. 
relationship between haemoglobin and clinical morbidity outcomes in COPD. Importantly, we note that haemoglobin is associated with morbidity in a nonlinear fashion, and we observe an optimal range of haemoglobin values. Finally, we identify haemoglobin derangement and gas transfer as distinct factors associated with morbidity. With very severe impairment in gas transfer, dyspnoea is high, and quality of life is poor, irrespective of haemoglobin. These novel results better characterise haemoglobin derangements in an epidemiologic study of stable COPD patients and further generate hypotheses regarding the relationship between haemoglobin and morbidity outcomes.

In the COPDGene cohort, we demonstrate a $14 \%$ prevalence of anaemia, and a $5 \%$ prevalence of polycythaemia, similar to prior studies in COPD [1, 2, 8-10, 35, 36]. In SPIROMICS, another large well-characterised cohort of COPD, Putcha et al. [10] described a prevalence of $7.5 \%$ for normocytic anaemia while our cohort had $\sim 13 \%$ with normocytic anaemia. This difference may be attributable to the enrichment for African-Americans in the COPDGene cohort; anaemia in COPD has been observed to be more common among African-Americans [10, 37, 38]. Prevalence of polycythaemia in our cohort is lower than prior estimates ranging from $6 \%$ to $8 \%$, but previous studies may have inadvertently been enriched for polycythaemia, with predominantly male and long-term oxygen therapy COPD patients [2, 8]. The presented results offer prevalence estimates in a general COPD population.

We observed microcytosis was present in a higher percentage of anaemic participants, while macrocytosis was more frequent in polycythaemic individuals, which is consistent with purported mechanisms of development of anaemia and polycythaemia in COPD. Systemic inflammation and impaired iron utilisation are implicated in development of anaemia in COPD [10, 11, 35, 39], which would present as either normocytic or microcytic anaemia. In concordance with this, higher CRP levels were noted among anaemic participants as compared to individuals with normal haemoglobin. Meanwhile, chronic hypoxia with subsequent erythropoietin stimulation leads to release of larger, immature red blood cells [40]. Consistent with this, polycythaemic patients had lower resting oxygen saturations and had more macrocytosis. Additionally, 56\% of polycythaemic participants were current smokers, and previous studies have described smoking-related elevations in carboxyhaemoglobin leading to development of polycythaemia [41, 42].

In analyses comparing anaemia and polycythaemia to a normal group, we identified significant independent associations between anaemia and morbidity across multiple domains. There have been numerous studies demonstrating the associations between anaemia and increased symptoms [2, 3, 8, 10], worse quality of life [2, 3, 10], decreased exercise performance [2, 3, 35], increased hospitalisations [5, 8, 36], and increased mortality [4, 8, 36, 43]. The presented findings confirm the association between anaemia and morbidity outcomes. Categorical analysis also identified no significant associations between polycythaemia and morbidity. CотE et al. [2] conducted a study of COPD participants recruited from a Veterans Affairs clinic and described no significant association between polycythaemia and functional status by mMRC and 6MWD. Our categorical results confirm these existing findings in the setting of a relatively low prevalence of polycythaemia compared with anaemia, and further explore the range of haemoglobin values in our continuous analyses.

We demonstrate novel findings of a nonlinear relationship between haemoglobin and morbidity across all domains. A nonlinear relationship between haemoglobin and morbidity has been described [10], but that study notably excluded those with polycythaemia or non-normocytic anaemia. Our results extend those findings by examining haemoglobin continuously across its entire observed range and demonstrating increased morbidity at either end of the haemoglobin spectrum, departing from the categorical analyses previously published [2,8] and described in this cohort. The findings of increased morbidity with haemoglobin values in the polycythaemia range, to our knowledge, have not been previously described, although studies preceding the routine use of long-term oxygen therapy describe improvement in exercise tolerance among COPD patients after phlebotomy to a haematocrit below 55\% [44, 45]. The discrepancy between this finding and the presented categorical analyses may be related to individuals with haemoglobin values close to the definition cut-off, minimising differences between those categorised as polycythaemic versus normal haemoglobin. The mechanism behind the increased morbidity associated with polycythaemia is not explored in this analysis, but may be related to untreated hypoxaemia, or active smoking, which generates carboxyhaemoglobin and can yield a functional anaemia that limits oxygen carrying capacity and thereby functional performance [41, 42].

Acknowledging the nonlinear relationship between haemoglobin and morbidity allows for identification and development of appropriate haemoglobin targets for interventions seeking to address anaemia in management of COPD. Such interventions include blood transfusions, iron administration, erythropoiesis-stimulating agent (ESA) administration, or novel small-molecule therapies. To date, few 
studies have been published evaluating the utility of treatment of anaemia in COPD. One small study noted that blood transfusions in stable COPD patients improved minute ventilation and reduced work of breathing [46]. Similarly, in a case series of five COPD patients in an intensive care unit setting, blood transfusions to an average haemoglobin concentration of $12 \mathrm{~g} \cdot \mathrm{dL}^{-1}$ were associated with more rapid weaning off a ventilator in COPD patients [47]. A small trial of intravenous iron therapy in patients with COPD noted improvements in dyspnoea and walk distance as compared with placebo [48]. Interestingly, the study was conducted in COPD patients irrespective of anaemia or iron deficiency, with an average haemoglobin level of $14.5 \mathrm{~g} \cdot \mathrm{dL}^{-1}$ and only $10 \%$ iron-deficient at baseline. Given our presented findings, the somewhat modest effects noted in this trial may be due to inappropriate selection of participants. Another small observational study of a combination of iron and ESA therapies in anaemic COPD patients noted an improvement in dyspnoea that correlated with the improvement in haemoglobin [49]. Finally, to date there are no studies in a COPD population of ESA therapy alone or small-molecule therapies that inhibit hypoxia-inducible factor, both of which are used or are being evaluated in CKD. The presented results offer information on appropriate selection of individuals who may benefit the most from management of haemoglobin derangements with such therapies, establishing a foundation for future studies in this arena.

Another key finding of this study was that both haemoglobin derangement and gas transfer impairment independently associated with morbidity outcomes, as demonstrated by a consistent relationship between haemoglobin and morbidity across various $D_{\text {LCO }}$ levels for all outcome domains except dyspnoea and quality of life. For these outcomes, beyond very severe gas transfer impairment, morbidity was high irrespective of haemoglobin level. The lack of effect modification between haemoglobin and $D_{\mathrm{LCO}}$ suggests that each measure is distinctly associated with morbidity. While potential mechanisms for the associations with morbidity were not assessed in this study, this generates hypotheses regarding $D_{\text {LCO }}$ as a reflection of parenchymal lung damage and haemoglobin possibly reflecting an inflammatory state in COPD. The relationships between $D_{\text {LCO }}$ and haemoglobin are complex, with likely variable associations across the spectrum of anaemia to polycythaemia, and the presented findings suggest that both $D_{\text {LCO }}$ and haemoglobin are relevant clinical factors to consider with respect to morbidity in COPD.

The present study is limited by the cross-sectional study design, precluding discussion of directionality of the associations identified, and self-report for many of the outcomes including COPD exacerbations. However, the consistency of the associations across multiple morbidity outcomes and with prior publications across multiple different cohorts suggests that these results are robust. Another key limitation of this study is incomplete characterisation of haemoglobin derangements. Microcytosis and macrocytosis prevalence were low among anaemic and polycythaemic individuals, limiting our ability to conduct analyses assessing effect modification of MCV across the spectrum of haemoglobin values. The lack of data on iron indices and erythropoietin levels limits discussion regarding mechanisms of haemoglobin derangements and the impact on morbidity. Similarly, measures of renal insufficiency would provide more accurate estimation of CKD in this population, a key comorbidity known to alter haemoglobin levels. However, as a surrogate, self-reported CKD and other comorbid conditions were included to limit confounding from multiple comorbid conditions including renal insufficiency. Finally, the present study, while suggestive of values that may define an optimal haemoglobin range for COPD patients, is a cross-sectional study without formal threshold analysis, in part due to the low prevalence of polycythaemia. Future studies across multiple large cohorts may better establish cut-off values of clinical relevance in a COPD population.

The presented findings demonstrate in a large cohort of COPD participants that haemoglobin derangements are prevalent and are independently associated with varying degrees of increased morbidity across the spectrum of observed haemoglobin values. Examination of haemoglobin as a continuous variable suggests that values in both the anaemia and polycythaemia ranges are associated with increased morbidity, and that there may be an optimal range of haemoglobin values that is relevant for a COPD population. These results offer support for future investigation of whether haemoglobin derangements drive morbidity or are markers of underlying disease severity and further exploration of factors that contribute to derangements in haemoglobin among this population. Additionally, these findings lay the foundation for research regarding potential therapeutic targeting of haemoglobin in management of COPD. In summary, this study establishes the association between haemoglobin and morbidity in COPD and supports consideration of haemoglobin measurement in evaluation of symptomatic individuals with COPD.

Author contributions: R.A. Wise contributed to the conception and design of the study, data analysis, interpretation, and preparation of this manuscript, and is the guarantor of this paper. A. Balasubramanian contributed to data analysis and interpretation, and manuscript writing. M.C. McCormack also contributed to data 
analysis and interpretation, and manuscript preparation and revision. R.J. Henderson contributed statistical support for analyses on the data. N.R. MacIntyre, R.L. Jensen, W.W. Stringer, C.P. Hersch, R.P. Bowler, R. Casaburi, M.K. Han, J. Porszasz and B.J. Make contributed to data interpretation and revision of the manuscript. All authors reviewed and approved the manuscript prior to submission for publication.

Conflict of interest: A. Balasubramanian has nothing to disclose. R.J. Henderson has nothing to disclose. N. Putcha reports grants from the NIH outside the submitted work. A. Fawzy has nothing to disclose. S. Raju has nothing to disclose. N.N. Hansel reports grants from the NIH and the COPD Foundation, grants and personal fees from AstraZeneca and GSK, grants from Boehringer Ingelheim, and personal fees from Mylan during the conduct of the study. N.R. MacIntyre reports personal fees from Inspirx, Ventec and Hillrom outside the submitted work. R.L. Jensen has nothing to disclose. G.L. Kinney has nothing to disclose. W.W. Stringer reports grants from AstraZeneca, and personal fees from Medical Director (Pulmonary Rehabilitation Program), Pulmonary and Critical Care Physician, and Medical Director (Blood Gas Laboratories), outside the submitted work. C.P. Hersh reports grants from the NHLBI during the conduct of the study; and grants from Bayer, Boehringer Ingelheim, Novartis and Vertex, outside the submitted work. R.P. Bowler has nothing to disclose. R. Casaburi has nothing to disclose. M.K. Han reports grants from the NHLBI during the conduct of the study; and personal fees from GSK, AZ, BI, Teva, Merck, Verona and Mylan, and research support from Sunovion and Novartis, outside the submitted work. J. Porszasz has nothing to disclose. B.J. Make reports grants, nonfinancial support and other support from AstraZeneca, nonfinancial support from Spiration, grants, nonfinancial support and other support from GlaxoSmithKline and Sunovion, other support from Mt Sinai, Web MD, National Jewish Health, Novartis, the American College of Chest Physicians, Projects in Knowledge and Hybrid Communications, grants from Pearl Research, other support from Medscape, Verona, Boehringer Ingelheim, Theravance and Ultimate Medical Academy, nonfinancial support and other support from Circassia, personal fees and nonfinancial support from Third Pole, nonfinancial support and other support from Phillips, other support from the Eastern Pulmonary Society, Catamount Medical, Science 24/7, Eastern VA Medical Center and Academy Continued Health Care Learning, grants from the NHLBI, personal fees from Takeda, and other support from Wolters Kluwer Health, outside the submitted work. M.C. McCormack has a patent with UpToDate with royalties paid, a patent with GSK pending, and a patent with Celgene pending. R.A. Wise reports personal fees from AstraZeneca/Medimmune/Pearl, grants and personal fees from Boehringer Ingelheim, personal fees from Contrafect, Roche-Genentech, Merck, Circassia, Pneuma, Verona, Mylan/Theravance and Propeller Health, grants from Sanofi-Aventis, personal fees from AbbVie, grants and personal fees from GSK, and personal fees from ChemRx, Kiniksa, Bristol Myers Squibb, Galderma, Kamada, Pulmonx, Kinevant and PureTech, outside the submitted work.

Support statement: This work was supported by NHLBI U01 HL089897 and U01 HL089856. The COPDGene study (NCT00608764) is also supported by the COPD Foundation through contributions made to an Industry Advisory Committee comprised of AstraZeneca, Boehringer Ingelheim, GlaxoSmithKline, Novartis and Sunovion. Funding information for this article has been deposited with the Crossref Funder Registry.

\section{References}

1 Sarkar M, Rajta PN, Khatana J. Anemia in chronic obstructive pulmonary disease: prevalence, pathogenesis, and potential impact. Lung India 2015; 32: 142-151.

2 Cote C, Zilberberg MD, Mody SH, et al. Haemoglobin level and its clinical impact in a cohort of patients with COPD. Eur Respir J 2007; 29: 923-929.

3 Ferrari M, Manea L, Anton K, et al. Anemia and hemoglobin serum levels are associated with exercise capacity and quality of life in chronic obstructive pulmonary disease. BMC Pulm Med 2015; 15: 58

4 Boutou AK, Karrar S, Hopkinson NS, et al. Anemia and survival in chronic obstructive pulmonary disease: a dichotomous rather than a continuous predictor. Respiration 2013; 85: 126-131.

5 Barba R, de Casasola GG, Marco J, et al. Anemia in chronic obstructive pulmonary disease: a readmission prognosis factor. Curr Med Res Opin 2012; 28: 617-622.

$6 \quad$ Kollert F, Tippelt A, Müller C, et al. Hemoglobin levels above anemia thresholds are maximally predictive for long-term survival in COPD with chronic respiratory failure. Respir Care 2013; 58: 1204-1212.

7 Toft-Petersen AP, Torp-Pedersen C, Weinreich UM, et al. Association between hemoglobin and prognosis in patients admitted to hospital for COPD. Int J Chron Obstruct Pulmon Dis 2016; 11: 2813-2820.

8 Chambellan A, Chailleux E, Similowski T. Prognostic value of the hematocrit in patients with severe COPD receiving long-term oxygen therapy. Chest 2005; 128: 1201-1208.

9 Shorr AF, Doyle J, Stern L, et al. Anemia in chronic obstructive pulmonary disease: epidemiology and economic implications. Curr Med Res Opin 2008; 24: 1123-1130.

10 Putcha N, Fawzy A, Paul GG, et al. Anemia and adverse outcomes in a chronic obstructive pulmonary disease population with a high burden of comorbidities. an analysis from SPIROMICS. Annals ATS 2018; 15: 710-717.

11 John M, Hoernig S, Doehner W, et al. Anemia and inflammation in COPD. Chest 2005; 127: 825-829. 
12 Vanfleteren LEGW, Spruit MA, Groenen M, et al. Clusters of comorbidities based on validated objective measurements and systemic inflammation in patients with chronic obstructive pulmonary disease. Am $J$ Respir Crit Care Med 2013; 187: 728-735.

13 Long term domiciliary oxygen therapy in chronic hypoxic cor pulmonale complicating chronic bronchitis and emphysema: report of the medical research council working party. Lancet 1981; 317: 681-686.

14 Continuous or nocturnal oxygen therapy in hypoxemic chronic obstructive lung disease: a clinical trial. nocturnal oxygen therapy trial group. Ann Intern Med 1980; 93: 391-398.

15 Regan EA, Hokanson JE, Murphy JR, et al. Genetic epidemiology of COPD (COPDGene) study design. COPD 2011; 7: 32-43.

16 WHO. Haemoglobin concentrations for the diagnosis of anaemia and assessment of severity. Vitamin and Mineral Nutrition Information System. Geneva, WHO, 2011. www.who.int/vmnis/indicators/haemoglobin.pdf

17 Barbui T, Thiele J, Gisslinger H, et al. The 2016 WHO classification and diagnostic criteria for myeloproliferative neoplasms: document summary and in-depth discussion. Blood Cancer J 2018; 8: 15.

18 Miller MR, Hankinson J, Brusasco V, et al. Standardisation of spirometry. Eur Respir J 2005; 26: 319-338.

19 Quanjer PH, Stanojevic S, Cole TJ, et al. Multi-ethnic reference values for spirometry for the 3-95-year age range: the global lung function 2012 equations. Eur Respir J 2012; 40: 1324-1343.

20 Stanojevic S, Grham B, Cooper B, et al. Global lung function initiative: Reference equations for the transfer factor for carbon monoxide (TLCO). Eur Respir J 2016; 48: OA283.

21 ATS statement: guidelines for the six-minute walk test. Am J Respir Crit Care Med 2002; 166: 111-117.

22 Han MK, Kazerooni EA, Lynch DA, et al. Chronic obstructive pulmonary disease exacerbations in the COPDGene study: associated radiologic phenotypes. Radiology 2011; 261: 274-282.

23 Jones PW, Harding G, Berry P, et al. Development and first validation of the COPD assessment test. Eur Respir J 2009; 34: 648-654.

24 Jones PW, Quirk FH, Baveystock CM. The St George's respiratory questionnaire. Respir Med 1991; 85: Suppl. B, 25-31, discussion 33-37.

25 Mahler DA, Ward J, Waterman LA, et al. Patient-reported dyspnea in COPD reliability and association with stage of disease. Chest 2009; 136: 1473-1479.

26 McHorney CA, Ware JE, Lu JF, et al. The MOS 36-item short-form health survey (SF-36): III. Tests of data quality, scaling assumptions, and reliability across diverse patient groups. Med Care 1994; 32: 40-66.

27 Singh D, Agusti A, Anzueto A, et al. Global strategy for the diagnosis, management, and prevention of chronic obstructive lung disease: the GOLD science committee report 2019. Eur Respir J 2019; 53: 1900164.

28 Royston P, Altman DG. Regression using fractional polynomials of continuous covariates: parsimonious parametric modelling. J R Stat Soc C 1994; 43: 429-467.

29 Royston P. Marginscontplot: plotting the marginal effects of continuous predictors. Stata J 2013; 13: 510-527.

30 Royston P, Sauerbrei W. Interactions. In: Multivariable Model-Building. John Wiley \& Sons, Ltd., 2008. pp. 151-182.

31 StataCorp. Stata Statistical Software: Release 15. College Station, TX, StataCorp LLC, 2017.

32 Jones PW. Interpreting thresholds for a clinically significant change in health status in asthma and COPD. Eur Respir J 2002; 19: 398-404.

33 Puhan MA, Chandra D, Mosenifar Z, et al. The minimal important difference of exercise tests in severe COPD. Eur Respir J 2011; 37: 784-790.

34 Holland AE, Hill CJ, Rasekaba T, et al. Updating the minimal important difference for six-minute walk distance in patients with chronic obstructive pulmonary disease. Arch Phys Med Rehabil 2010; 91: 221-225.

35 Boutou AK, Stanopoulos I, Pitsiou GG, et al. Anemia of chronic disease in chronic obstructive pulmonary disease: a case-control study of cardiopulmonary exercise responses. Respiration 2011; 82: 237-245.

36 Similowski T, Agustí A, MacNee W, et al. The potential impact of anaemia of chronic disease in COPD. Eur Respir J 2006; 27: 390-396.

37 Lee H, Shin SH, Gu S, et al. Racial differences in comorbidity profile among patients with chronic obstructive pulmonary disease. BMC Med 2018; 16: 178

38 Krishnan G, Grant BJ, Muti PC, et al. Association between anemia and quality of life in a population sample of individuals with chronic obstructive pulmonary disease. BMC Pulm Med 2006; 6: 23.

39 Duru S, Bilgin E, Ardiç S. Hepcidin: a useful marker in chronic obstructive pulmonary disease. Ann Thorac Med 2012; 7: 31-35.

40 Moore-Gillon JC, Treacher DF, Gaminara EJ, et al. Intermittent hypoxia in patients with unexplained polycythaemia. Br Med J (Clin Res Ed) 1986; 293: 588-590.

41 Smith JR, Landaw SA. Smokers' polycythemia. N Engl J Med 1978; 298: 6-10.

42 Aitchison R, Russell N. Smoking: a major cause of polycythaemia. J R Soc Med 1988; 81: 89-91.

43 Martinez-Rivera C, Portillo K, Muñoz-Ferrer A, et al. Anemia is a mortality predictor in hospitalized patients for COPD exacerbation. COPD 2012; 9: 243-250.

44 Chetty KG, Brown SE, Light RW. Improved exercise tolerance of the polycythemic lung patient following phlebotomy. Am J Med 1983; 74: 415-420. 
45 Harrison BDW, Davis JN, Madgwick R, et al. The effects of therapeutic decrease in packed cell volume on the responses to exercise of patients with polycythaemia secondary to lung disease. Clin Sci Mol Med 1973; 45: 833-847.

46 Schonhofer B, Wenzel M, Geibel M, et al. Blood transfusion and lung function in chronically anemic patients with severe chronic obstructive pulmonary disease. Crit Care Med 1998; 26: 1824-1828.

47 Schönhofer B, Böhrer H, Köhler D. Blood transfusion facilitating difficult weaning from the ventilator. Anaesthesia 1998; 53: 181-184.

48 Santer P, McGahey A, Frise MC, et al. Intravenous iron and chronic obstructive pulmonary disease: a randomised controlled trial. BMJ Open Respir Res 2020; 7: e000577.

49 Silverberg DS, Mor R, Weu MT, et al. Anemia and iron deficiency in COPD patients: prevalence and the effects of correction of the anemia with erythropoiesis stimulating agents and intravenous iron. BMC Pulm Med 2014; 14: 24 . 